\title{
EFFICIENT DAMAGE ASSESSMENT AND REPAIR IN RESILIENT DISTRIBUTED DATABASE SYSTEMS
}

\author{
Peng Liu and Xu Hao
}

\begin{abstract}
Preventive measures sometimes fail to defect malicious attacks. With cyber attacks on data-intensive applications becoming an ever more serious threat, intrusion tolerant database systems are a significant concern. The main objective of intrusion tolerant database systems is to detect attacks, and to assess and repair the damage caused by the attacks in a timely manner such that the database will not be damaged to such a degree that is unacceptable or useless. This paper focuses on efficient damage assessment and repair in resilient distributed database systems. The complexity of distributed database systems caused by data partition, distributed transaction processing, and failures makes damage assessment and repair much more challenging than in centralized database systems. This paper identifies the key challenges and presents an efficient algorithm for distributed damage assessment and repair.
\end{abstract}

\section{INTRODUCTION}

Recently, people have seen more and more successful attacks on data-intensive applications, especially web-based e-business and e-government applications. Expanding cyber attacks pressure database management systems to not only prevent unauthorized access, but also tolerate intrusions. However, traditional database security techniques such as authorization $[8,9]$, inference control [1], multilevel secure databases [19], and multilevel secure transaction processing [4], are very limited to handle successful attacks, or intrusions. This indicates that intrusion tolerant database systems that can detect intrusions, isolate attacks, contain, assess, and repair the damage caused by intrusions can become a significant concern.

The focus of this paper is on a critical aspect of intrusion tolerant database systems, namely, damage assessment and repair. The primary goal of the attacks on a database, in most cases, is to damage the data stored in the database (in an undetectable way) and use the damaged data to mislead people to make wrong decisions. Compared with the attack of completely shutting down a database system, damaging a set of data items (or objects) can be much more dangerous, 
not only because they are more difficult to detect, but also because the damage can spread seriously in a database through benign operations. In particular, any benign transaction that reads a corrupted data item can help spread the damage accidentally. The main objective of an intrusion tolerant database system is to guarantee that damage spreading is (dynamically) controlled in such a way that the database will not be damaged to a degree that is unacceptable or useless. Being a key technique to control damage spreading, damage assessment and repair is a significant concern.

One key technique for building intrusion tolerant database systems is intrusion detection, which has attracted many researchers [14, 17]. Effective damage assessment and repair cannot be achieved without effective intrusion detection because intrusion detection can tell which users or operations can cause damage, however, intrusion detection makes the system attack-aware but not attack-resilient, that is, intrusion detection itself cannot maintain the integrity and availability of the database in face of attacks.

This paper focuses on the techniques to assess and repair the damage caused in a distributed database. Distributed databases are widely used in a variety of real world data intensive applications, and we need efficient damage assessment and repair algorithms to make them resilient to attacks. Several algorithms are developed to assess and repair the damage caused in a centralized database $[3,18,11]$, however, the complexity of distributed database systems caused by data partition, distributed transaction processing, and failures suggests that they cannot be used in distributed database systems and new distributed damage assessment and repair algorithms are needed. First, since data are partitioned and stored on multiple sites, damage assessment and repair must be performed at multiple sites. Second, since one distributed transaction may access data stored at multiple sites, damage assessment, which is now based on the affecting relationships among distributed transactions, needs to coordinate and synthesize the affecting relationships among the subtransactions at each site. Third, distributed damage assessment and repair need to tolerate not only site failures but also communication failures.

Finally, it should be noticed that although our approach focuses on surviving attacks by malicious transactions, database intrusion tolerance can also be enforced at some lower levels such as OS level. However, although transaction level methods cannot handle OS level attacks, it is shown that in many applications where attacks are enforced mainly through malicious transactions transaction level methods can tolerate intrusions in a much more effective and efficient way. Moreover, it is shown that OS level intrusion tolerance techniques such as those proposed in $[14,13,15,5]$, can be directly integrated into a transaction level intrusion tolerance framework to complement it with the ability to tolerate OS level attacks. 


\subsection{Prior Work}

Database recovery mechanisms are not designed to deal with malicious attacks. Undo and redo operations can only be performed on uncommitted or active transactions, and the durability property ensures that traditional recovery mechanisms never undo committed transactions [6]. However, the fact that a transaction commits does not guarantee that its effects are desirable. Specifically, a committed transaction may reflect malicious activity.

Although our assessment and repair model is related to the notion of cascading abort [6], cascading aborts only capture the read-from relation between active transactions, and in standard recovery approaches cascading aborts are avoided by requiring transactions to read only committed data [10].

\subsection{Our Approach and Contribution}

In this paper, we extend centralized damage assessment and repair techniques to distributed systems, in particular, we present an efficient on-the-fly transactionoriented algorithm to assess and repair the damage caused in a distributed database. The algorithm has the following properties: (1) It is completely distributed, thus it has no single point of failures and it can tolerate malicious attacks on end systems and communication links. (2) It is on-the-fly. New transactions are continuously executed during the damage assessment and repair process. (3) It is efficient. Damage assessment and repair are performed, in parallel, at each participating site. (4) It works seamlessly with a commercial DDBMS, thus it can be directly applied to build resilient distributed database applications. (5) It provides transparent damage assessment and repair.

The rest of the paper is organized as follows. In Section 2, we present the model for distributed damage assessment and repair. We present the damage assessment and repair algorithm in Section 3. In Section 4, we address some performance issues of the algorithm. We conclude the paper in Section 5.

\section{THE MODEL}

\subsection{Distributed Database Model}

A distributed database is a set of data items (or objects) stored across a set of sites connected by a network. Each data item is stored at one and only site. A distributed transaction is a partial order of read and write operations on these data items that either commits or aborts. Two operations conflict if one is write. Two transactions conflict if they have conflicting operations. The execution of a set of distributed transactions is modeled by a (distributed) history. The model of a distributed transaction execution is such that there is one process, called the coordinator, that is connected to the user application and a set of other processes, called the subordinates. Transactions are assumed to have globally unique identifiers. The processes are assumed to have globally unique 
identifiers, which also indicate the sites (or locations) of the corresponding processes. For simplicity, we assume that each site participating in a distributed transaction has only one process of that transaction.

We assume the distributed database system supports transaction serializability and uses the two-phase locking (2PL) protocol. We assume the two-phase commit (2PC) protocol is used to guarantee the atomicity of distributed transactions, despite intermittent site or communication link failures. We assume the standard 2PC protocol is used here [6]. To save space, the details about how $2 \mathrm{PC}$ handles the log are not reviewed. It should be noticed that although our damage assessment and repair algorithm is specified based on the way log records are maintained, our algorithm can be easily extended to support other $2 \mathrm{PC}$ protocols such as presumed abort (PA) and presumed commit (PC) [16].

\subsection{Damage Assessment and Repair Model}

Our idea of damage assessment and repair is that only the malicious transactions and the innocent transactions affected by a malicious transaction, directly or indirectly, can cause damage and should be repaired. In order to make this idea more tangible, we first formally define the affecting relationship among transactions. Since damage can only be caused by committed transactions, our definitions care about only committed transactions. We denote the set of committed malicious transactions in a history by $\mathbf{B}=\left\{B_{1}, B_{2}, \ldots, B_{m}\right\}$. We denote the set of committed innocent transactions by $\mathbf{G}=\left\{G_{1}, G_{2}, \ldots, G_{n}\right\}$.

Definition 1 At a site, a committed subtransaction $T_{j k}$ is dependent upon committed subtransaction $T_{i l}$ if there exists a data item $x$ stored at the site such that $T_{j k}$ reads $x$ after $T_{i l}$ has updated $x$, and no other committed subtransactions update $x$ between the time $T_{i l}$ updates $x$ and $T_{j k}$ reads $x$.

Definition 2 A committed distributed transaction $T_{j}$ is dependent upon committed distributed transaction $T_{i}$ in a history if one of $T_{j}$ 's subtransactions is dependent upon one of $T_{i}$ 's subtransactions.

Definition 3 In a history, a committed distributed transaction $T_{i}$ affects committed distributed transaction $T_{j}$ if the ordered pair $\left(T_{j}, T_{i}\right)$ is in the transitive closure of the dependent upon relation.

It is clear that the notation "affect" captures both direct and indirect affecting relationships. To illustrate, consider the following history over $\left(B_{1}, G_{1}, G_{2}\right)$, which are assumed to be executed on two sites. Here $r$ means a read, $w$ means a write, and $c$ means a commit.

At site $A: H_{A}=r_{B_{1 A}}[x] w_{B_{1 A}}[x] c_{B_{1 A}} r_{G_{1 A}}[x] r_{G_{1 A}}[y] w_{G_{1 A}}[y] c_{G_{1 A}} r_{G_{2 A}}[z] w_{G_{2 A}}[z] c_{G_{2 A}}$ At site $B: H_{B}=r_{B_{1 B}}[u] w_{B_{1 B}}[u] c_{B_{1 B}} r_{G_{1 B}}[v] w_{G_{1 B}}[v] c_{G_{1 B}} r_{G_{2 B}}[v] r_{G_{2 B}}[w] w_{G_{2 B}}[w] c_{G_{2 B}}$ 
Based on $H_{A}$, we find that $G_{1 A}$ is dependent upon $B_{1 A}$, so $G_{1}$ is affected by $B_{1}$, and $x$ and $y$ are damaged. We find that $G_{2 A}$ is neither dependent upon $B_{1 A}$ nor $G_{1 A}$. However, this does not ensure that $G_{2}$ will not be affected by $B_{1}$. Based on $H_{B}$, we find that $G_{2 B}$ is dependent upon $G_{1 B}$ (although $G_{2 B}$ is not dependent upon $B_{1 B}$ ), so $G_{2}$ is dependent upon $G_{1}$. Since $G_{1}$ is dependent upon $B_{1}$, so $\left(G_{2}, B_{1}\right)$ is in the transitive closure of the dependent upon relation. Therefore, $G_{2}$ is actually affected by $B_{1}$ and $w$ is damaged.

\section{THE ALGORITHM}

We use two types of processes to do distributed damage assessment and repair (DAR): a Local DAR Manager on each site, and a Local DAR Executor on each site. The idea is to do distributed damage assessment and repair through local operations. At a site, the Local DAR Executor is responsible for scanning the local $\log$ to (a) identify the subtransactions that are affected by a malicious transaction and (b) compose the corresponding cleaning subtransactions for these subtransactions; the Local DAR Manager is responsible for coordinating the DAR process of (only) the distributed transactions that had their coordinators at the site and are affected by a malicious transaction. In particular, for each such transaction $T_{i}$, the Local DAR Manager will create the coordinator for $T_{i}$ 's cleaning transaction, and instruct (only) the Local DAR Executors at the sites where $T_{i}$ 's subordinates were located to assess the damage spread by $T_{i}$ and to repair $T_{i}$. Cleaning transactions are executed just like an normal transaction.

The example described in Section 2 shows that in order to capture every transaction affected by a malicious transaction, global coordination among sites is necessary, since a transaction affected by a malicious transaction may be found unaffected at some of its subordinates' sites if only local information is used. Our algorithm does global coordination by making the Local DAR Managers and Executors collaborating with each other. In particular, after $B$ is identified, it will be first sent, in parallel, to the Local DAR Managers at the sites where B's coordinators were located. These Local DAR Managers then send an ASSESS message to ask each of the Local DAR Executors at the sites where B's subordinates are located to assess the damage caused by $\mathbf{B}$ on their local data. The identifier of each affected (sub)transaction found by the Local DAR Executors then will be sent, in parallel, to the Local DAR Managers at the sites where the coordinators of these affected transactions were located, which will then send an ASSESS message to ask each of the Local DAR Executors at the sites where the other subordinates of these affected transactions were located to assess the damage indirectly caused by $\mathbf{B}$. This kind of interaction between Local DAR managers and Executors will be continuously performed until all the damage is located. 
The repair process is relatively simpler. The damage caused by each malicious or affected distributed transaction is repaired by a distributed cleaning transaction. The Local DAR Managers are responsible for creating the coordinators for these cleaning transactions. The Local DAR Executors are responsible for composing the cleaning subtransactions that comprise each cleaning transaction. It should be noticed that it is not guaranteed that a cleaning subtransaction will really clean each item it wants to clean. Sometimes, an item can be really cleaned after several cleaning subtransactions have updated the item. The reason is that cleaning subtransactions are composed only based on local information, which may not be complete.

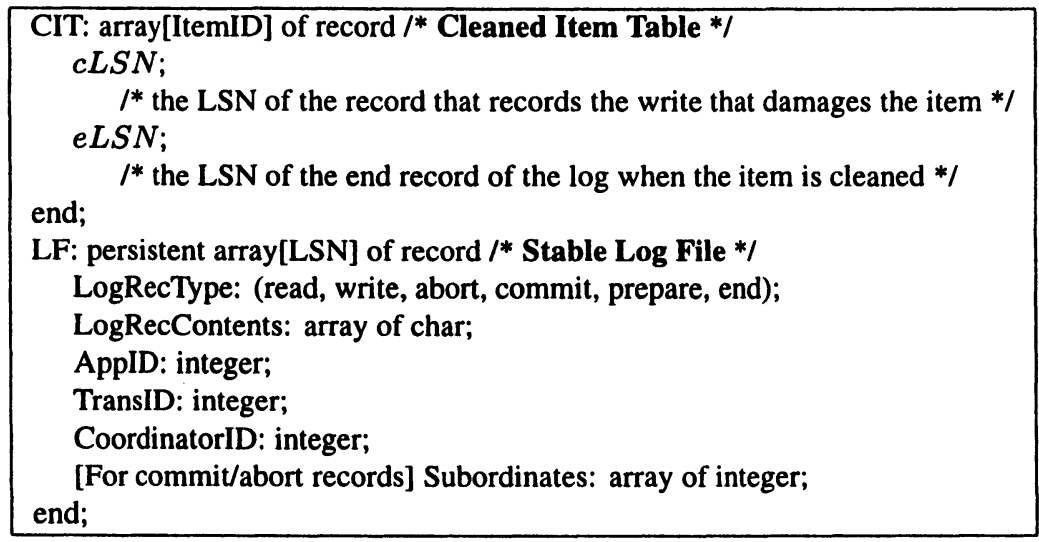

Figure 1. Major Data Structures

\subsection{Data Structures}

Our algorithm is based on the transaction log maintained on each site. We assume every read (operation) is recorded in a log. Although this is not the case for most commercial distributed database systems, [3] shows that (a) in addition to logging reads there are several other ways to collect reads such as extracting read sets from transaction profiles, and (b) the algorithms based on in-log reads can be easily extended to use the reads maintained outside the logs. We assume each log has six types of records, namely, read, write, abort, commit, prepare, and end records. The other possible types of records such as savepoints and checkpoints are not considered here because they are irrelevant. We assume the log records at each site contain the type (read, prepare, commit, etc.) of the record, the identifier of the application, the identifier of the transaction, the identifier of the coordinator, the identities of the subordinates in the case of the commit/abort records written by the coordinator [16].

In addition to logs, our algorithm uses the following data structure: 
- an Cleaned Item Table $(C I T)$ at each site that contains the items that have been 'cleaned' at the site. Each item in this table is tagged with two numbers: $c L S N$, which indicates when the item is damaged, and $e L S N$, which indicates when the item is cleaned.

These data structures are described in Figure 1. Here ItemID means the identifier of a data item. $L S N$, means $\log$ serial number, a unique number associated with each $\log$ record. $L S N$ becomes larger as the log grows.

\subsection{Damage Assessment and Repair Under Normal Operation}

First, we describe the algorithm without considering failures. The algorithm is as follows. Note that here for clarity we assume during the DAR process no new malicious transactions will be added to $\mathbf{B}$, although the algorithm can be easily extended to support newly identified malicious transactions.

\footnotetext{
Algorithm 1 Distributed On-the-fly Damage Assessment and Repair Algorithm

Input: $B$, the logs

Output: when the DAR process terminates, any prefix $H_{p}$ of the history including the point where the process terminates results in the state that would have been produced by $H_{p}^{\prime}$, where $H_{p}^{\prime}$ is $H_{p}$ with all the transactions in $\mathbf{B}$ or affected by $\mathbf{B}$ removed

Initialization:

- make $P$, a pointer, pointed to the header of the $\log$, which is before any log records;

- assess_list $:=\{\}$, to_assess $:=\{\}$, undo_list $:=\{\}$, tmp_undo_list $:=\{\}$, dirty_item_set $:=$ \{\}, tmp_item_set $:=\{\}$, submitted_item_set $:=\{\} ; I^{*}$ See comment $\mathbf{A} *$ I
}

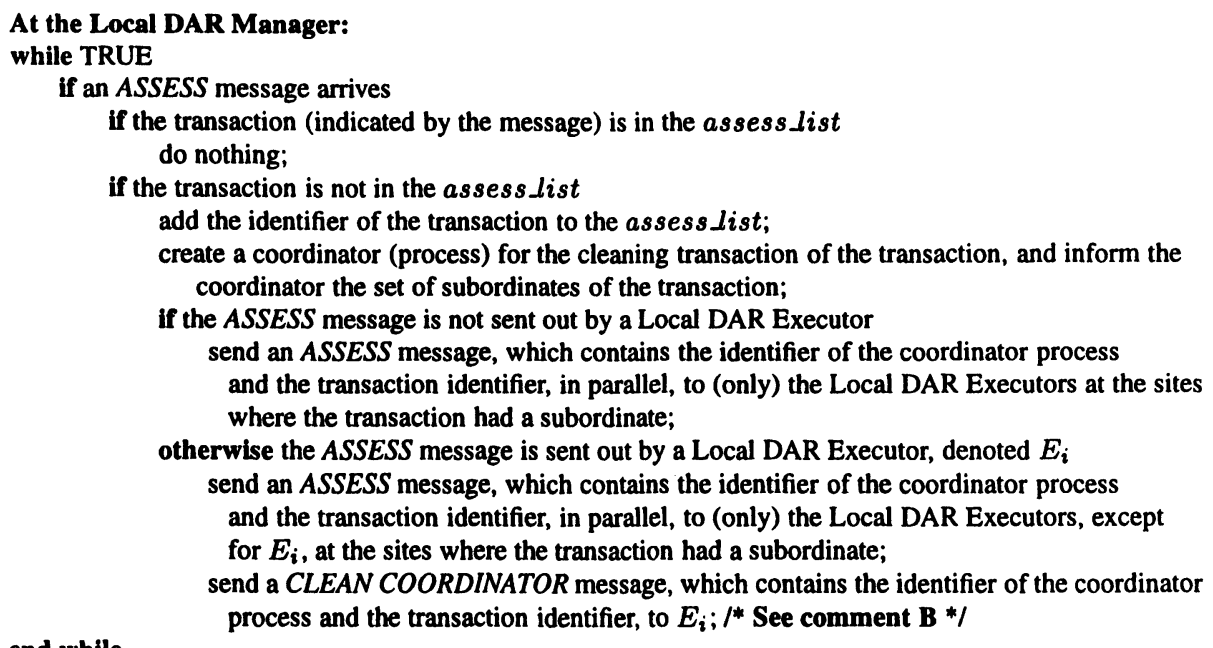

end while

At the Local DAR Executor:

while TRUE

if a CLEAN COORDINATOR message arrives

associate the identifier of the coordinator with the transaction kept in the undo_list ;

if an SUCCESS message (sent from a coordinator of a cleaning transaction) arrives 
move each item $x$ that has been cleaned from the dirty_item_set to the $C I T$ table, and

associate $x$ with the $L S N$ of the current end of the log (namely $x . e L S N$ );

remove $x$ from the submitted_item_set;

if an ASSESS message arrives

if this is the first ASSESS message

make $P$ pointed to the first log record of the transaction;

elseif the transaction is not in the undo_list /* See comment $C *$ /

add the transaction (indicated by the message), together with the coordinator identifier, to

to.assess;

if the first $\log$ record of the transaction is before the record pointed by $P$ /* See comment $D * /$

if the transaction is not in the tmp_undo_list

make $P$ pointed to the first $\log$ record of the transaction;

else remove the transaction from the tmp_undo_list, and move the items that are written

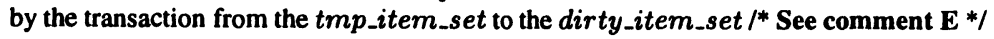

if $P$ points to a record and the termination conditions do not hold $/ *$ See comment $F *$ I

Scan the log entry pointed by $P$ :

if the entry is for a cleaning transaction

skip it;

elseif the entry is for a transaction $T_{i}$ in to_assess

if the entry is a write record $\left[T_{i}, x, v_{1}, v_{2}\right]$

if $x$ is not in the $C I T$ table

add $x$, together with the $L S N$ of the write record (denoted $x . c L S N$ ), to the dirty_item_set;

elseif $x . e L S N<w . L S N$ or $w . L S N<x . c L S N /$ * See comment G */

add $(x, x . c L S N)$ to the dirty_item_set;

elseif the entry is a commit record $\left[T_{i}\right.$, commit]

compose the cleaning subtransaction for $T_{i} ; / *$ See comment $\mathbf{H} *$ /

send the composed cleaning subtransaction to its coordinator (the identifier of

the coordinator can be found in to_assess);

elseif the entry is of other types

skip it;

else

case the entry is a write record $\left[T_{i}, x, v_{1}, v_{2}\right]$

if $x$ is not in the $C I T$ table

add $(x, x . c L S N)$ to the tmp_item_set;

elseif $x . e L S N<w . L S N$ or $w . L S N<x . c L S N$

add $(x, x . c L S N)$ to the tmp_item_set;

case the entry is a read record $\left[T_{i}, x\right]$

if $x$ is in the dirty_item_set and r.LSN>x.cLSN/* See comment $1 *$ I add $T_{i}$ to the tmp_undo_list;

elseif $x$ is in the $C I T$ table and $r . L S N<x . e L S N / *$ See comment $\mathrm{J} *$ / add $T_{i}$ to the tmp_undo_list;

case the entry is an abort record $\left[T_{i}\right.$, abort $]$

delete all the data items of $T_{i}$ from the tmp_item_set;

if $T_{i}$ is in the tmp_undo_list, remove it;

case the entry is a commit record [ $T_{i}$, commit]

if $T_{i}$ is in the tmp_undo_list

move all the items of $T_{i}$ from the tmp_item_set to the dirty_item_set;

move $T_{i}$ from the tmp_undo_list to the undo_list;

send an ASSESS message, which contains the identifier of the transaction, to the

Local DAR Manager at the site where the coordinator of $T_{i}$ was located; compose the cleaning subtransaction for $T_{i}$;

send the composed cleaning subtransaction to its coordinator (the identifier of the coordinator can be found in the undo_list);

else delete all the items of $T_{i}$ from the tmp_item_set;

case the entry is of other types

skip it; 
end while $P=P+1$;

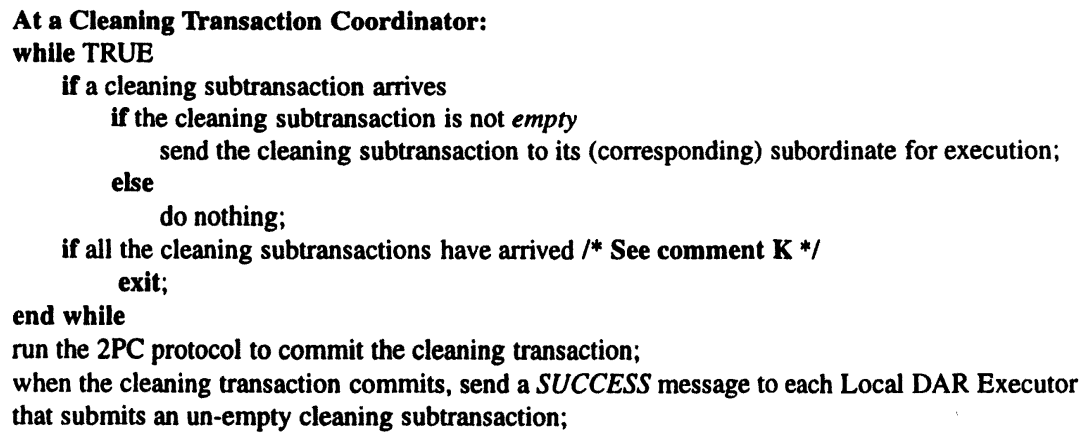

\section{Comments}

A. Inside a Local DAR Manager, the assess_list contains (the identifiers of) the transactions that have been reported to the Local DAR Manager. Inside a Local DAR Executor, the submitted_item_set contains the items that are being cleaned but still not cleaned. The to_assess contains the set of transactions in $\mathbf{B}$ that has a subordinate at the site, and the set of innocent transactions that had a subordinate at the site, are affected by $\mathbf{B}$ but are not identified by the Local DAR Executor. The undo_list contains the transactions affected by $B$ and identified by the Local DAR Executor. The $t m p \_u n d o \_l i s t$ contains the transactions that are found affected but may abort later on. The dirty_item_set contains the damaged items. The $t m p \_i t e m_{-}$set contains the items that could have been damaged. When a Local DAR Executor scans an innocent transaction, it does not know whether or not the transaction will commit later on. Even if the transaction reads a damaged item, it will cause no damage if it aborts later on. Therefore, we use the tmp_item_set and the tmp_undo_list to contain the information about which transactions may have caused damage, and which items may have been damaged. In this way, we need not to re-collect this information when a transaction commits.

B. Although $E_{i}$ does not need the ASSESS message, $E_{i}$ still needs the identifier of the coordinator process to repair the damage caused by $T_{i}$ at $E_{i}$ 's site.

C. If the transaction $T$ is in the undo_list, it is already found affected at the site, and an ASSESS message $M$ has already been sent to a Local DAR Manager. The reason that this site still receives an ASSESS message for $T$ is because the Local DAR Manager receives another ASSESS message for $T$ before $M$.

D. Given the transaction is not in the undo_list, if it is not in the tmp_undo_list, it is still not found affected at the site. Hence if its first $\log$ record is before $P$, then the Local DAR Executor had not assessed the damage caused by the transaction when it was scanned, so we need to rescan the transaction and its following transactions.

E. Although the transaction is in the tmp_undo_list, since the transaction is already added to to_assess, the items of the transaction kept in the tmp_item_set will no longer be handled. Hence we need to move them now to the dirty_item_set.

F. Since the algorithm allows users to continuously execute new transactions as the damaged items are identified and cleaned, new transactions can spread damage if they read a damaged but still unidentified item. So we face two critical questions: (1) Will the DAR process terminate? (2) If the DAR process terminates, can we detect the termination? 
Fortunately, [3] has answered these two questions in the context of centralized intrusion tolerant database systems. Here we give similar answers for distributed systems. First, when the damage spreading speed is quicker than the damage repair speed, the DAR process may never terminate. When the damage repair speed is quicker, the DAR process will terminate. Second, under the following conditions we can ensure that the DAR process terminates: (1) the cleaning transaction for every (reported) malicious transaction has been committed; (2) at each site, dirty_item_set $=\emptyset$; (3) at each site, $t m p \_$undo_list $=\emptyset$, and $\forall x \in C I T, x . e L S N<l . L S N$. Here, $l . L S N$ denotes the $L S N$ of the next log record for the Local DAR Executor to scan. (4) At each site, the Local DAR Manager has no ASSESS message to send, and the Local DAR Executor has no ASSESS message to respond. Condition 1 ensures that no malicious transaction is needed to repair. Condition 2 ensures that every identified damaged item is cleaned. Conditions 3 and 4 ensure that further scans will not identify any new damage. Please refer to [3] for more details about termination detection.

G. $w . L S N$ denotes the $L S N$ of the write record. If $w . L S N>x . e L S N, x$ is cleaned before the write operation, therefore, the write damages $x$ again. If $w . L S N<x . c L S N$, this write is before the write that has been cleaned, so this write damages $x$ before the cleaned write. So $x$ is not really cleaned (by previous cleaning transactions).

H. The composing algorithm works as follows: for each item $x$ written by $T_{i}$,

- if $x$ is in the CIT table or the submitted_item_set, and $x . c L S N$ is larger than both the $c L S N$ value of $x$ kept in the clean_item_set (if any) and the $c L S N$ value of $x$ kept in the submitted_item_set (if any), then add no cleaning operation of $x$ to the cleaning subtransaction of $T_{i}$ (because $x$ is already or to be 'better' cleaned);

- otherwise, add a write operation, which restores $x$ to its previous value (before the write), to the cleaning subtransaction of $T_{i}$, and add $(x, x . c L S N)$ to the submitted_item_set.

It should be noticed that under some situations, for example, when $T_{i}$ at the site is readonly, or when all the items written by $T_{i}$ have been 'better' cleaned, an empty cleaning subtransaction may be composed.

I. $r . L S N$ denotes the $L S N$ of the read record. If $r . L S N>x . c L S N, x$ is read after $x$ is damaged.

J. If $r . L S N<x . e L S N, x$ is still not cleaned when $x$ is read.

K. Since the coordinator was informed by its Local DAR Manager the list of subordinates of the transaction to clean, it knows the sites from which a cleaning subtransaction is expected.

\subsubsection{Analysis of the Algorithm}

Our algorithm has the following characteristics:

Claim 1. An ASSESS message for transaction $T$ is sent out by a Local Executor only if $T$ is affected by a malicious transaction.

Claim 2. For each innocent transaction affected by a malicious transaction (in B), the Local DAR Executor at every site where the transaction had a subordinate will assess and repair the damage caused by the transaction. 
Claim 3. When the DAR process terminates, every item that is updated by a transaction in B or affected by B will be restored to the value before the item is damaged.

Claim 4. If the DAR process terminates, the algorithm can detect the termination.

Based on these characteristics, the correctness of our algorithm can be specified as follows.

Theorem 1 Algorithm 1 is correct in the sense that (a) if the DAR process terminates, the algorithm can detect the termination and stop scanning the logs, and (b) when the DAR process terminates, any prefix $H_{p}$ of the history including the point where the DAR process terminates results in the state that would have been produced by $H_{p}^{\prime}$, where $H_{p}^{\prime}$ is $H_{p}$ with all the transactions in $\mathbf{B}$ or affected by $\mathbf{B}$ removed.

\subsection{Damage Assessment and Repair Under Failures and System Attacks}

Let us now consider site and communication failures. We not only consider the failures caused by accidental errors, but also consider the failures caused by intentional attacks. In particular, during a DAR process, we assume a site can crash due to errors, we also assume a site can be attacked to crash. We assume due to errors or attacks, a message can be corrupted, lost (or dropped), faked, or replayed. We also assume a communication link can be broken for a while due to errors or attacks. We finally assume all failed sites ultimately recover, and all broken communication links are ultimately reconnected.

The impact of failures is two folds: First, corrupted, lost, faked, or replayed messages among the DAR Managers, the DAR Executors, and the cleaning transaction coordinators can cause a transaction to be assessed and repaired multiple times, can cause an innocent unaffected transaction to be "repaired" (rolled back), and can cause some malicious or affected transactions to stay at large. Second, crashed sites or broken communication links can disable some message interactions among the DAR Managers, the DAR Executors, and the cleaning transaction coordinators. In particular, when a site is unreachable during a period of time, its DAR Manager cannot receive (send) ASSESS messages from (to) DAR Executors at other sites; its DAR Executor cannot send ASSESS messages to DAR Managers at other sites; and its cleaning transaction coordinators cannot execute cleaning transactions at other sites. As a result, some information about affected transactions (generated at one site) cannot be distributed to other relevant sites in a timely manner, and some damaged items 
at one site can only be identified (and repaired) after another site becomes reachable.

The algorithm proposed in Section 3.2 can be easily extended to tolerate failures. First, our discussion in next section, namely Section 4, indicates that standard secure communication techniques such as IPSEC can be used to ensure that the messages between a Local DAR Manager and a Local DAR Executor or between a Local DAR Executor and a cleaning transaction coordinator will not be changed, dropped, faked, or replayed. Second, in order to tolerate failed sites and broken communication links, we assume that at each active site a recovery process exists and that it processes all messages from recovery processes at other sites and handles all the recovery tasks assigned by the Local DAR Manager and the Local DAR Executor. We assume that cleaning transaction coordinators (at each site) use standard distributed database fault tolerance services to execute cleaning transactions.

At an active site, when the Local DAR Manager or the Local DAR Executor find that a message cannot be sent out, they will forward the message, together with the information about who should receive the message, to the local recovery process, then "forget" the message and continue working. It is the responsibility of the recovery process to resend these unsuccessful messages when they can be sent out. When a site recovers, its recovery process will broadcast (or multicast) a RESTART message to all the other recovery processes, which will then send out all the messages for the recovered site.

Since a site may crash at any point of time, we need to periodically keep state information for the DAR components so that they need not restart the DAR process from the very beginning when a site recovers. In particular, at each site we assume a message log is kept for each DAR component, including the Local DAR Manager, the Local DAR Executor, the local recovery process, and the local cleaning transaction coordinators. Whenever a DAR component receives a message, the message will be first logged. In addition, we assume each DAR component periodically takes a checkpoint of its current state. When a message for a DAR component is logged, the message is tagged with the latest checkpoint taken by the component. In this way, when a site recovers, each DAR component, starting from its latest checkpoint, knows exactly which messages should be reprocessed. Moreover, when a message for the Executor is logged, it will also be tagged with the $L S N$ of the $\log$ record that is currently scanned. In this way, the Executor can synchronize messages and scan operations.

The above method can ensure that every message will be processed, but it can neither ensure that every message will be received only once nor ensure that messages will be received in their original (sending-out) orders. Fortunately, we can use the message log kept for each DAR component to filter off the messages that had been received. Moreover, our algorithm can tolerate misordered messages. For each cleaning transaction coordinator, the arriving order 
of cleaning subtransactions does not matter. For each DAR Manager, if two ASSESS messages do not arrive in the original order, they will be processed in the wrong order. As a result, at a site even if $T_{i}$ is dependent upon $T_{j}$, the Local DAR Executor could receive the ASSESS message for $T_{i}$ before that for $T_{j}$ (Note that this can happen even if each Local DAR Manager and Local DAR Executor receives ASSESS messages in their original orders). Fortunately, the correctness of our Executor algorithm, which is based on the (absolute) time order indicated by log records, is not affected by the order of arriving messages.

Since our DAR system is basically nondeterministic (See that a Local DAR Executor could do different things after receiving an ASSESS message and before receiving another ASSESS message), distributed fault tolerance techniques for deterministic systems such as message logging cannot be directly used $[7,20,2]$. Our approach, which can be viewed as a pessimistic message logging approach tailored based on the semantics of DAR processes, is simple, does not need global coordination, and can tolerate nondeterministic events.

\section{PERFORMANCE ISSUES}

Providing intrusion tolerance usually will affect the system performance. Therefore, a tradeoff between performance and intrusion tolerance should be (and has to be) made in most intrusion tolerant systems. Fortunately, our algorithm typically has very little impact on the performance of distributed transaction processing. The DAR components are completely separated from transaction processing components. In most cases when only few transactions are malicious the number of transactions affected by a malicious transaction is very small compared with the total number of transactions. We have done some performance study in centralized intrusion tolerant database systems using simulated data [12], the results show that on average only $2.72 \%$ transactions are affected by a malicious transaction. Although executing cleaning transactions makes the system busier, the number of cleaning transactions is typically very small compared with the total number of user transactions, and the Damage Confinement Manager can help prevent cleaning transactions from conflicting with user transactions. Our performance study in centralized intrusion tolerant database systems shows that on average only $0.32 \%$ items are damaged [12]. Although the DAR messages make the network busier, the number of DAR messages is typically very small compared with the number of transaction processing messages.

\section{CONCLUSION}

We have analyzed the problem of and proposed an efficient completelydistributed algorithm for damage assessment and repair in secure and resilient distributed database systems. We believe we have accomplished an impor- 
tant step towards providing "data integrity" guarantees to arbitrary distributed database applications in face of attacks.

\section{Acknowledgments}

Liu was supported by the Defense Advanced Research Projects Agency (DARPA) and Air Force Research Laboratory, Air Force Material Command, USAF, under agreement number F30602-00-2-0575.

\section{References}

[1] M. R. Adam. Security-Control Methods for Statistical Database: A Comparative Study. ACM Computing Surveys, 21(4), 1989.

[2] L. Alvisi and K. Marzullo. Message logging: Pessimistic, optimistic, causal and optimal. IEEE Transactions on Software Engineering, 24(2):149-159, 1998.

[3] P. Ammann, S. Jajodia, and P. Liu. Recovery from malicious transactions. IEEE Transactions on Knowledge and Data Engineering, 2001. To appear.

[4] V. Atluri, S. Jajodia, and B. George. Multilevel Secure Transaction Processing. Kluwer Academic Publishers, 1999.

[5] D. Barbara, R. Goel, and S. Jajodia. Using checksums to detect data corruption. In Proceedings of the 2000 International Conference on Extending Data Base Technology, Mar 2000.

[6] P. A. Bernstein, V. Hadzilacos, and N. Goodman. Concurrency Control and Recovery in Database Systems. Addison-Wesley, Reading, MA, 1987.

[7] A. Borg, J. Baumbach, and S. Glazer. A message system supporting fault tolerance. Operating System Review, 17(5):90-99, October 1983.

[8] P. P. Griffiths and B. W. Wade. An Authorization Mechanism for a Relational Database System. ACM Transactions on Database Systems, 1(3):242-255, September 1976.

[9] S. Jajodia, P. Samarati, V. S. Subrahmanian, and E. Bertino. A unified framework for enforcing multiple access control policies. In Proceedings of ACM SIGMOD International Conference on Management of Data, pages 474-485, May 1997.

[10] H.F. Korth, E. Levy, and A. Silberschatz. A formal approach to recovery by compensating transactions. In Proceedings of the International Conference on Very Large Databases, pages 95-106, Brisbane, Australia, 1990.

[11] P. Liu, P. Ammann, and S. Jajodia. Rewriting histories: Recovery from malicious transactions. Distributed and Parallel Databases, 8(1):7-40, 2000.

[12] P. Luenam and P. Liu. Odam: An on-the-fly damage assessment and repair system for commercial database applications. In Proc. 15th IFIP WG11.3 Working Conference on Database and Application Security, 2001.

[13] Teresa Lunt and Catherine McCollum. Intrusion detection and response research at DARPA. Technical report, The MITRE Corporation, McLean, VA, 1998.

[14] T.F. Lunt. A Survey of Intrusion Detection Techniques. Computers \& Security, 12(4):405418, June 1993.

[15] J. McDermott and D. Goldschlag. Towards a model of storage jamming. In Proceedings of the IEEE Computer Security Foundations Workshop, pages 176-185, Kenmare, Ireland, June 1996.

[16] C. Mohan, B. Lindsay, and R. Obermarck. Transaction management in the $\mathrm{r}^{*}$ distributed database management system. ACM Transaction on Database Systems, 11(4):378-396, 1986. 
[17] B. Mukherjee, L. T. Heberlein, and K.N. Levitt. Network intrusion detection. IEEE Network, pages 26-41, June 1994.

[18] B. Panda and J. Giordano. Reconstructing the database after electronic attacks. In Proceedings of the 12th IFIP 11.3 Working Conference on Database Security, Greece, Italy, July 1998.

[19] R. Sandhu and F. Chen. The multilevel relational (mlr) data model. ACM Transactions on Information and Systems Security, 1(1), 1998.

[20] R. E. Strom and S. Yemini. Optimistic recovery in distributed systems. ACM Transaction on Computer System, 3(3):204-226, August 1985. 